\title{
Software Management in Product Structure
}

\author{
Gülden Şenaltun and Can Cangelir \\ Turkish Aerospace Industries Inc., Ankara, Turkey 06980 \\ \{gsenaltun, ccangelir\}atai.com.tr
}

\begin{abstract}
In order to develop high quality products with lower cost and in a shorter time according to the conventional methods, Concurrent Engineering techniques should be applied on projects. Concurrent Engineering discipline requires a collaborative way of working of relevant departments in a factory. With a view to manage all product data collaboratively and to give related information to different departments, product structure should be constituted in a proper way by leading of Concurrent Engineering Department.

In this paper, definition and content of product structure are explained. Product structure content is detailed regarding to aircraft structure which has different sections including all type of detailed parts like harness, equipment and structural parts. Moreover, management of product structure in lifecycle of an aircraft is considered. This topic is studied by examining different phases of an aircraft lifecycle in which the concept of product structure is explained. Furthermore, importance of using PLM (Product Lifecycle Management) tools for management of product structure is mentioned. With the help of PLM tools usage, management of software in product structure is studied by different methods. Advantages and disadvantages of these methods are examined and the most efficient method is proposed for software management.
\end{abstract}

Keywords: Concurrent Engineering, Product Structure, Software Management, Concurrent Engineering Department.

\section{Introduction}

As mentioned in The 14th International Conference on Machine Design and Production, Concurrent Engineering is a systematic approach to create a product design that considers in parallel all elements of the product lifecycle from the concept phase of the design to release of the product, and in so doing, defines the product, its manufacturing process, and all other required lifecycle processes such as logistic support. In this manner, Product Structure is used by the help of PLM tools [1]. Product Structure is constructed at the beginning of product lifecyle and Concurrent Engineering Department leads to this construction.

In this paper, product structure is defined and the necesstiy of using PLM (Poduct Lifecycle Management) tools are explained. Detailed information about product structure is given and the relation between product structure and product lifecyle is explained. Software management in product structure is an issue to control all aircraft data in one tool. Software management should be done in PLM tools and this 
management is done with configuration control by product structure. In this paper, management of software in product structure is studied. Three different methods are examined for software management and advantages/disadvantages are studied for these methods.

\section{Product Structure and PLM Tool}

\subsection{Definition of Product Structure}

According to Military Handbook (MIL-HDBK) 61B; "Product structure derived from the functional analysis and allocation process of system engineering, may be depicted graphically as a tree structure or as an indentured listing” [2].

Dolezal states that product structure is composed of main and sub-components in a hierarchical way. It refers to system architecture, internal structure, and relationship of system components and associated configuration documentation. [3] According to Peltonen, product structure explains the fact how the product is divided into components, which are in turn divided into subcomponents, etc. [4].

Product structure consists of different type of parts in aerospace industry such as structural parts, systems, equipments, harness, softwares, etc. Product structure brings all different kinds of parts together in an organizational way. Product structure keeps not only the 3D model of parts, but also contains meta data which gives all information about parts like material information, manufacturing process, etc.

Product structure lives during all product lifecylce. Aircraft design has different phases with different needs. To manage design data more accurately, different product structures are constructed during a lifecycle of the aircraft. There are no manufacturing activities up to development phase. Therefore, product structure is constructed according to design organization and activities. This product structure is called "Definition Product Structure" which represents the functional breakdown of the aircraft. It is called an "Engineering View" by Dolezal [3]. With starting of development phase, manufacturing activities are started and product structure should be reconstructed. This is "Development Product Structure" where components are divided according to manufacturing activities and manufacturing planning. This product structure represents the way the aircraft is produced and integrated. It is called "Manufacturing View" of the aircraft by Dolezal [3].

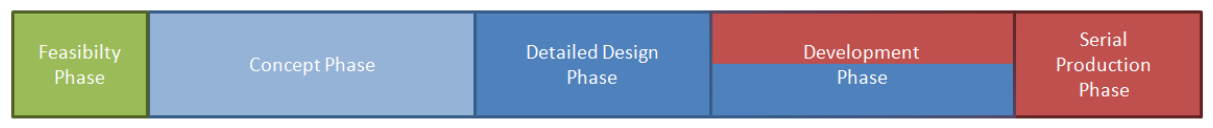

\section{Definition Product Structure}

Develpment Product Structure

Fig. 1. Phases of aircraft design and type of used Product Structure 
Using different product structures gives chance to define the whole aircraft according to the needs of all different departments, like design, manufacturing, procurement, supportability, maintainability, etc. in the organization. It is a master information source for industrialization, maintenance, test and marketing. Figure 2 is used by Dolezal to explain how product structure serves the different departments [3].

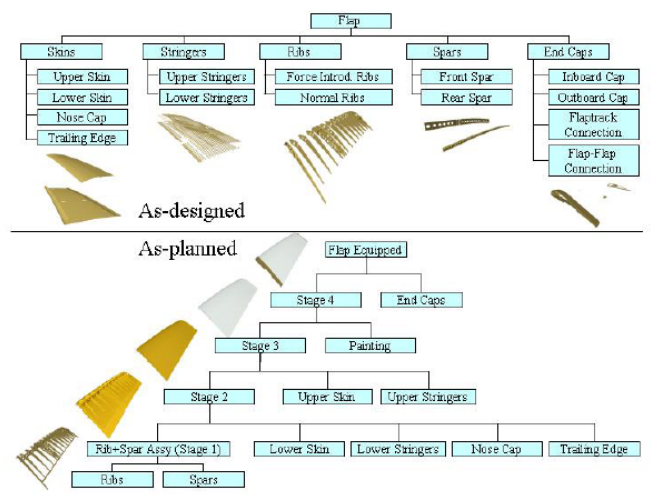

Fig. 2. Different views of Product Structure [3]

For configuration management, product should be broken down into manageable elements. These elements are called Configuration Item (CI). CI is the point where the configuration status is managed. The technical response of CI is Design Solutions (DS). DS provides all necessary elements to build the solution. Different products need different technical solutions because of requirements. The same function/assembly is controlled with one CI in the product structure. In this case, different DSs are needed and this is controlled with effectivity given by MSN (Manufacturing Serial Number). Figure 3 is an example for different DSs for one CI.

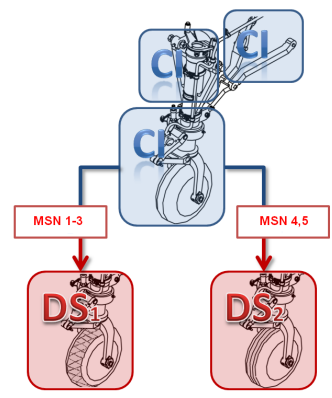

Fig. 3. CI-DS Relation

Product Structure and BOM (Bill of Material) are different terms used in this paper. BOM is used to refer to the list of parts used in one assembly. Product structure is used to refer the whole structure constructed for an aircraft. As an example, the BOM is used for DS to mention parts and assemblies which DS consists of. 


\subsection{Usage of PLM Tools}

PLM tools are used to manage product structure and to store all data in a single database; hence every user can reach this information by local computers. Product structure keeps latest design information and by the help of PLM tools every user can reach the latest design information at the same time.

In PLM tools, each detailed part/assembly is represented as an item in the product structure with part number and part description. Detailed information about parts, like 3D model and meta data is kept under item revisions. Item revisions are released in PLM tools and changes are controlled on item revisions. If there is a need to update on part, after release of latest revision, item is revised again and new revision is updated according to new requirements. Latest item revisions are linked to product structure.

To construct and maintain product structure properly, different item types and different roles are used in PLM tools. Every technical personnel is responsible for its own item type and, he/she could not change other design data. This shows the importance of using PLM tools in the organization to manage design data in a more suitable and protected way.

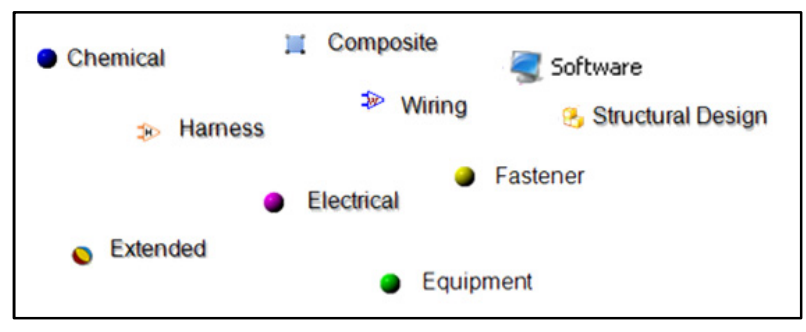

Fig. 4. Example of Different Item Types

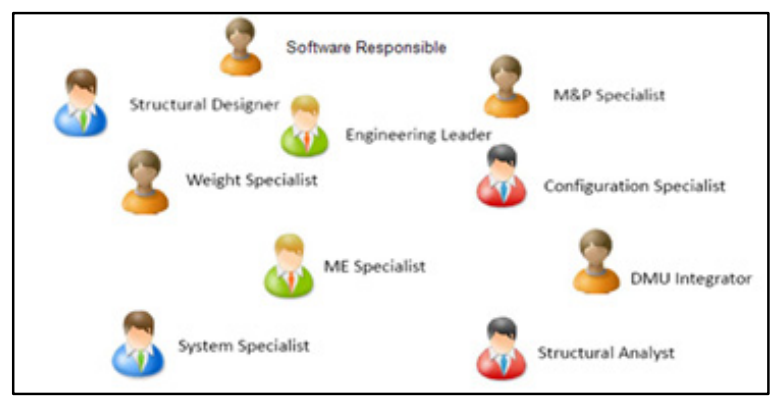

Fig. 5. Example of Different Roles

There are two different options to construct product structure in PLM tools. These are "Precise" and "Imprecise" type of product structure. In precise product structure, detailed parts item revisions are linked to the assembly's item revisions. In this option, if the detailed part item is revised, all assemblies above that part should be revised. In imprecise product structure, detailed part items are linked to assembly's item 
revision. In this option, last revision of part is linked to assembly and assemblies shouldn't be revised because of new part revision. Precise product structure keeps the history of assemblies in order to know which detail part revision is used for which assembly revision. This is important, when organization working style needs this information for manufacturing side. In this study, precise product structures are used.

\section{Software Management}

Software is a programme that provides the instructions for telling equipment what to do and how to do it. This programme consists of a package of codes developed in computer science. This code package is called as "Software Code". For each software, there is a unique number called "Software Number" like an item number. Software development is tracked by version information and every version is released.

In this paper, it is considered that software development is not managed in PLM tools. For development, special tools are used. On the other hand, equipments are managed in PLM tools with all their information. Software number and version should be linked to equipment information and to aircraft effectivity. To manage this link, software number and its version should be managed in PLM tools without its code information.

Software development is a complex process and this development starts at the definition phase and it proceeds during the development phase. During this development, software version is upgraded before activation of software for aircraft. Since only effectivity information of software and equipment relations are kept in PLM tools for software management, software version number that is used for aircraft is loaded to PLM tools and it is linked with effectivity and equipment in development phase. Since software are managed in PLM tools at development phase and Development Product Structure, there is no need to manage software in Definition Product Structure.

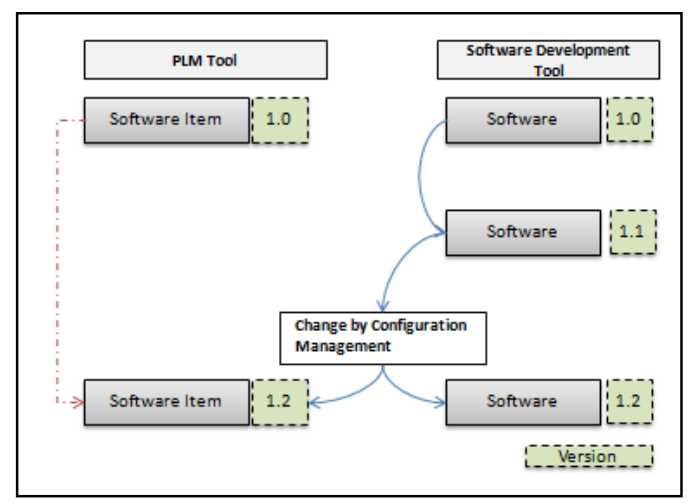

Fig. 6. PLM Tools and Software Developments Tools Relation

Change management is done for the software design management side and for the PLM tools side. For the software management, software code is reviewed for change and "Software Configuration Group" leads and controls the change management process. For the PLM tools, change management is controlled by "Configuration 
Management Group", who is responsible for the aircraft configuration, and software version and its relation with equipment and aircraft effectivity is considered. There is a change procedure in PLM tools to manage change management done by Configuration Management Group.

In some cases, equipment effectivity and software effectivity are not the same. Same equipment can be used with different softwares for different aircrafts. This difference is mentioned with terms "Equipment Effectivity" and "Software Effectivity".

Sofware and equipment relation with effectivity information shoul be listed from PLM tools. This is done with the reporting option of PLM tools. Special reports should be taken from PLM tools. Figure 7 is an example for a report taken from PLM Tool.

\begin{tabular}{|l|c|l|c|}
\hline $\begin{array}{l}\text { Equipment } \\
\text { Number }\end{array}$ & $\begin{array}{l}\text { Equipment } \\
\text { Effectivity }\end{array}$ & $\begin{array}{l}\text { Software } \\
\text { Number }\end{array}$ & $\begin{array}{l}\text { Software } \\
\text { Effectivity }\end{array}$ \\
\hline Equipment 1 & $1-6$ & Software 1 & $1-3$ \\
\hline Equipment 2 & 7-on & Software 3 & $3-6$ \\
Equipmet 3 & 11-on & Software 2 & 7-on \\
\hline
\end{tabular}

Fig. 7. Report format taken from PLM Tool

There are three different methods to manage software number and its version information in PLM tools and to link this information with an equipment and aircraft effectivity.

\subsection{Method 1}

In this method, software is kept as a meta data of its relevant equipment as seen in Figure 8 .

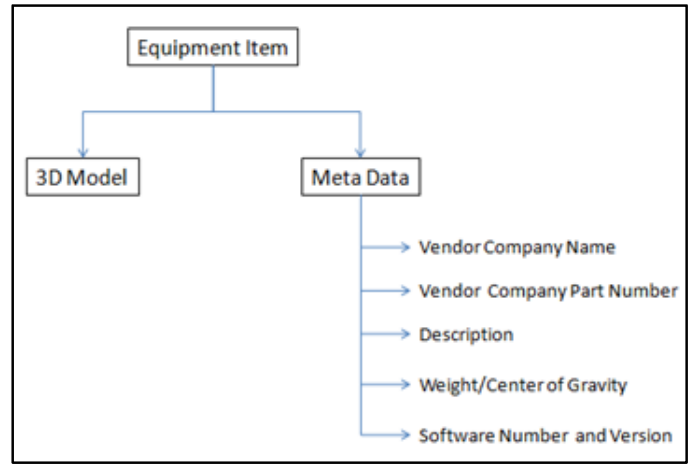

Fig. 8. Equipment Data in PLM tools

Equipment is managed under System DS (Figure 9) and effectivity is managed on this System DS. There is no separate information about software effectivity in product structure. Software effectivity is the same with equipment of which software is kept as meta 
data. If software effectivity is different for aircraft which has the same equipment, this fact should be controlled on System DS. System DS is multiplied according to software effectivity. Moreover, same equipment with different softwares for different aircrafts couldn't be managed with the same equipment number, since their meta data is different. These are the disadvantages of this software management method.

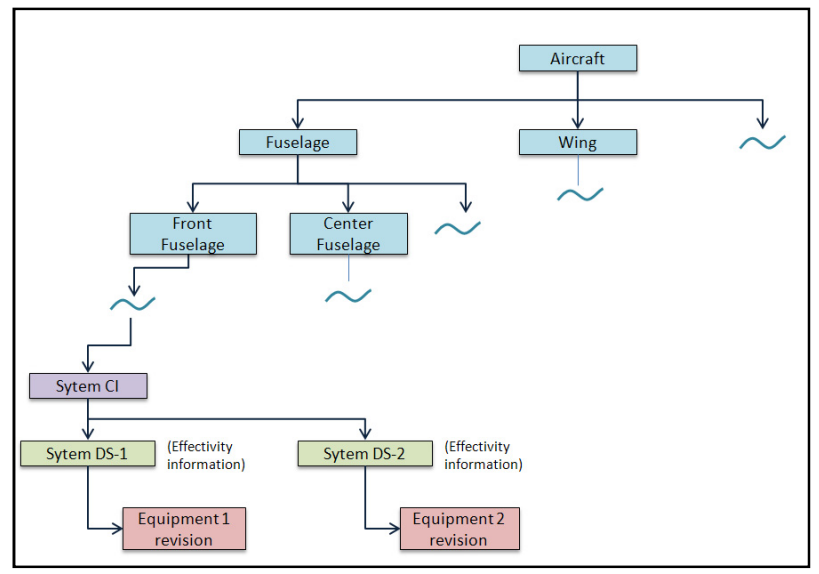

Fig. 9. Product Structure for Method 1

Another disadvantage of this method is the fact that version change of software affects equipment revision. To apply version change, the equipment should be revised and new version should be written as a meta data for new the revision of equipment. Like a first disadvantage, this causes extra work for system specialist responsible for equipment. This brings an extra change management work for the equipment revision in PLM tools, also.

Report preparation could be considered as an advantage of this method. Report preparation is simple since link between software version and equipment is kept as meta data. Equipment effectivity and software effectivity are the same. Moreover, System DS effectivity is valid for an equipment and software. This effectivity management is simple for Configuration Management Group. This is an another advantage of this method.

\subsection{Method 2}

In this method, software is managed as an item, Software Item, in PLM tools. Its version information is kept as item revision. There are equipment and software revisions in System DS's BOM as seen in Figure 10. Effectivity is controlled in System DS; hence equipment effectivity and software effectivity should be the same.

Report preparation is simple in this method. System DS BOM has equipment and software version with effectivity information and BOM report is enough for all needed information about software management. This is an advantage of the second method.

In this method, software version change affects the System DS revision. To connect the last version of software to System DS, System DS should be revised Moreover, equipment should be checked if version change affects equipment or not. 


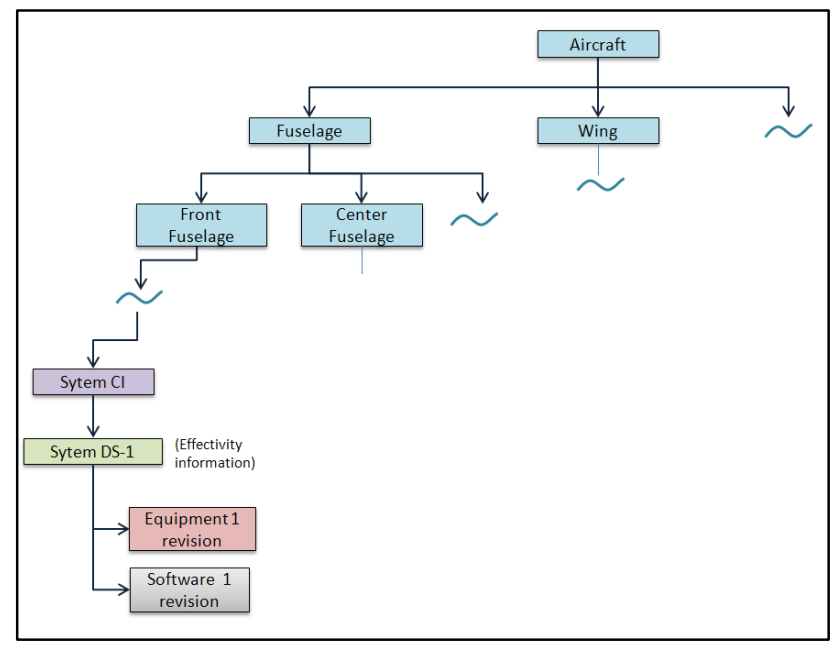

Fig. 10. Product Structure for Method 2

This effect is considered in change procedure leaded by Configuration Management Group. The "System Specialist" role in PLM tools, is responsible for System DS and equipment. This change brings extra work to the system specialist such as revising System DS and equipment. Extra work for the system specialist should be considered as one of the disadvantages.

Another disadvantage of this method is about effectivity management. This method is suitable for the case where the equipment effectivity and software effectivity are same for all aircraft. If same equipment has different softwares for different aircrafts, system DS should be prepared according to software effectivity as shown in Figure 11. This brings the system specialist extra work. Moreover, controlling System DSs will be more complex for configuration management group.

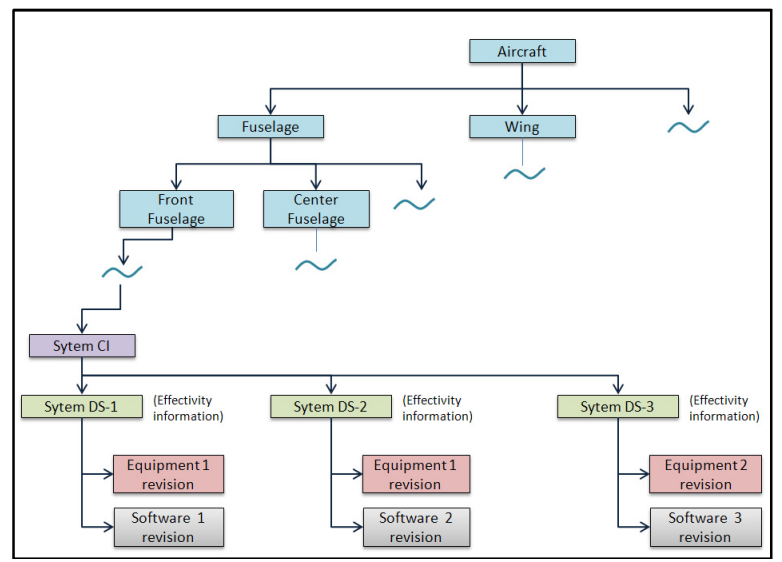

Fig. 11. Product Structure for different software with different effectivity 


\section{3 $\quad$ Method 3}

In this method software is managed as an item in PLM tools like in Method 2. Software item is linked to Equipment revision in PLM tools. This link is independent from software version. System DS is composed of only equipment revision. To control software effectivity there is another branch under "Aircraft", at the top of the product structure on Software DS apart from equipment. (Figure 12)

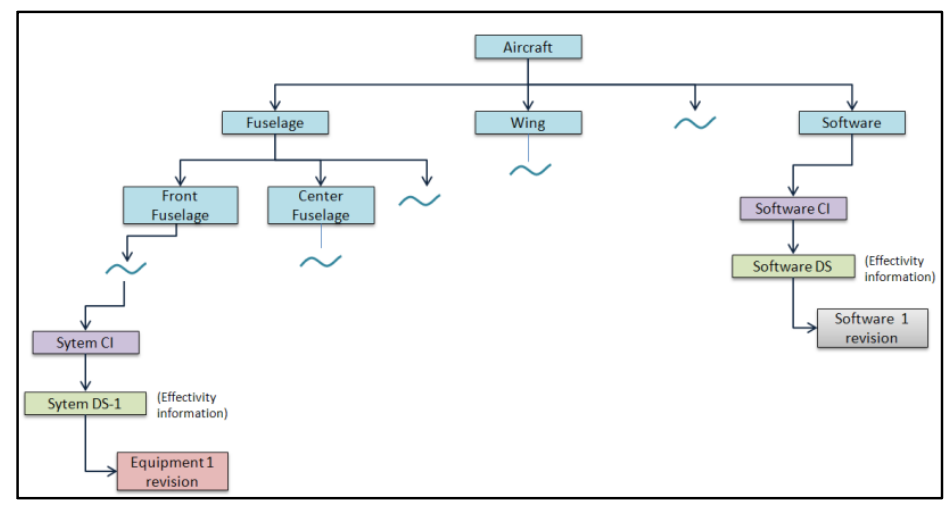

Fig. 12. Product Structure for Method 3

In this method, equipment is not linked to software version and the last version of the software is considered to be efficient for aircraft. Equipment is not directly affected from software version changes and this makes management of equipments and System DS easier. If software version change affects the equipment 3D model or meta data, this effect is controlled by the configuration management group in software change management procedure. If there is an effect, equipment will be revised. This brings a flexibility to control equipment revision and there is no extra work explained in Method 1 and Method 2 for the system specialist in every software version change. Elimination of direct effect of software change to equipment and System DS is the most important advantage of this method.

Moreover, there is no link between software effectivity and system DS. Software effectivity is controlled on Sofware DS. If there is an effectivity change for software, system DS won't be revised because of this change. elimination of system DS revisions is an another advantage of this method,

Reporting can be considered as a disadvantage, since there is no link between equipment and software version, also effectivities of equipment and software is managed in separate branches. Taking all information from separate places needs extra customization for reporting in PLM tools.

\section{Conclusion}

In this paper software management in PLM tools for aircraft industry has been studied. For this study, the concept of product structure is explained. Different types of 
product structures and relevant terms are explained. The need for PLM tools has been examined to construct product and manage it in the whole aircraft lifecycle. Usage of PLM tools and PLM tools' properties are discussed.

Three different methods for software management are explained with their advantages and disadvantages for software management in PLM tools.

Method 1 and Method 2 are simple in terms of management of effectivity and reporting. It should be possible that different disciplines work separately in PLM tools. However, in these two methods, the system specialist and the software responsible could not work separately. Software version and effectivity changes affect equipment and System DS.

Reporting for Method 3 is more complex than Method 1 and Method 2. This complex work is done once and then used for all projects in PLM tools. Different disciplines, system and software, are able to work separately with this method. Management of effectivity is more complex than the other two methods; however, this could be solved by PLM tools.

Comparing these three methods, management software as an item and management of software effectivity in a separate branch, Method 3, is the most suitable one.

\section{References}

1. Military handbook 61B, Configuration Management Guidance, pp. 5-5 (2002)

2. Cangelir, C.: Construction of Product Definition Data for Concurrent Engineering. In: The 14th International Conference on Machine Design and Production, June 29-July 02 (2010)

3. Dolezal, W.: Success Factors for DMU in Complex Aerospace Product Development, Technische Universität München (November 2007)

4. Peltonen, H.: Concepts and an Implementation for Product Data Management. Acta Polytechnica Scandinavica, Mathematics and Computing Series No. 105, Espoo (2000) 